\title{
ABSOLUTE NORMS ON VECTOR LATTICES
}

\author{
by J. M. BORWEIN and D. T. YOST
}

(Received 2nd December 1983)

\section{Introduction}

Recall that a norm $\|\cdot\|$ on a vector lattice $E$ is absolute if $\||x|\|=\|x\|$ for all $x \in E$; and monotone if $\|x\| \leqq\|y\|$ whenever $0 \leqq x \leqq y$. If the norm is both absolute and monotone, it is called a Riesz norm. It is easy to show that a norm is Riesz if and only if $\|x\| \leqq\|y\|$ whenever $|x| \leqq|y|$. A Banach lattice is a vector lattice equipped with a complete Riesz norm.

This note is motivated by the observation that any absolute norm on $\mathbb{P}^{n}$ (equipped with the usual order) is already monotone, hence a Riesz norm [1, Theorem 2]. This follows from the fact that if $|x| \leqq|y|$ then $x$ lies in the convex hull of $\{z:|z|=|y|\}$. The authors find it surprising that this problem has not been considered in a more general setting.

If any vector space (other than $\mathbb{R}$ ) is equipped with the lexicographic ordering induced by some basis, then $|x| \in\{-x, x\}$ for every $x$. Thus every norm is absolute, although no norm is monotone. The lexicographic ordering is bad in the sense that it is not compatible with the topology of $\mathbb{R}^{n}$. Order bounded sets may be topologically unbounded, the positive cone is not closed and the lattice operations are not continuous.

It turns out that boundedness of order intervals is the right property to ensure that every absolute norm is monotone. Having a closed cone or a complete norm does not help, as the theorems and examples will show.

The converse problem-must a monotone norm be absolute?-is much simpler. There are many norms on $\mathbb{R}^{2}$ (with the usual order) which are monotone but not absolute. More generally, let $E$ be a Banach lattice, and $f$ a positive functional on $E$ which is not a lattice homomorphism. If we define $\||x|\|=\|x\|+|f(x)|$ then $\|\cdot \mid\|$ is a monotone norm which is not absolute.

\section{Absolute norms on vector lattices}

We begin by proving the result mentioned in the introduction.

Theorem 1. Let $E$ be a vector lattice, equipped with an absolute norm $\|\cdot\|$, such that every order interval is norm bounded. Then $\|\cdot\|$ is a Riesz norm.

Proof. Suppose $y \geqq 0$ and $\|y\| \leqq 1$. We must show that the interval $[0, y]$ lies in the ball $B=\{x:\|x\| \leqq 1\}$. 
Let $I=\left\{\lambda \in \mathbb{R}^{+}:[-y, y] \subseteq \lambda B\right\}$. Since $[-y, y]$ is bounded, $I$ is not empty. We show that if $i \in I$, then $\frac{1}{2}(\lambda+1) \in I$.

Given $x \in[-y, y]$ and $\lambda \in I$, put $z=2|x|-y$. Clearly $-y \leqq z \leqq y$, so $z \in \lambda B$. Hence $\|\cdot x\|=\||\cdot|\|=\frac{1}{2}\|y+z\| \leqq \frac{1}{2}(\lambda+1)$. Thus $\frac{1}{2}(\lambda+1) \in I$.

Since $I$ is a closed subset of $\mathbb{R}$, it now follows that $1 \in I$. Thus $[0, y] \subseteq[-y, y] \subseteq B$.

We remark that the proof of Theorem 1 works just as well for a seminorm instead of a norm. Thus a topological vector space, lattice-ordered by some normal cone, is a topological vector lattice iff the topology is determined by a family of absolute seminorms.

Corollary 2. Let $E$ be a Banach lattice under some norm. Then every smaller absolute norm is a Riesz norm.

We have not been able to find a property which is dual to absoluteness of the norm. Nonetheless, the following is true.

Corollary 3. Let $E$ be a Banach lattice, $\|\cdot\|$ an equivalent norm for E. Suppose $\||f|\|=\|f\|$ for every $f \in E^{*}$. Then $E$ and $E^{*}$ are Banach lattices under $\|\cdot\|$.

Proof. Clearly every order interval in $E^{*}$ is bounded, so $\|\cdot\|$ is a Riesz norm for $E^{*}$.

Examples 2, 3 and 4 will show that the dual of lattice order need not be a lattice order, since the dual cone need not be generating.

Our first example shows that we do need to assume boundedness of intervals in Theorem 1.

Example 1. Let $E$ be the space of absolutely continuous functions on $[-1,1]$, whose derivatives are essentially bounded. With the pointwise ordering, $E$ is a vector lattice. Equipped with the norm $\|f\|=|f(0)|+$ ess.sup $\left|f^{\prime}\right|, E$ is a Banach space. An elementary argument shows that $\left.|| f\right|^{\prime}|=| f^{\prime} \mid$ almost everywhere (in fact at all points of differentiability of $|f|$ ) and so the norm is absolute. However, it is easy to see that the interval $[0,1]$ is not a bounded subset of $E$, although it is linearly bounded. Thus $E$ has no equivalent Riesz norm.

Although the positive cone is closed, the lattice operations in $E$ are not continuous. To see this, define $f, f_{n} \in E$ by $f(t)=t$ and $f_{n}(t)=t-1 / n$. Then $f_{n} \rightarrow f$ but

$$
\left\|f_{n}^{+}-f^{+}\right\|=1+1 / n
$$

In view of Example 1, it is appropriate to investigate continuity of the lattice operations.

Theorem 4. Let $E$ be a vector lattice. A norm on $E$ is a Riesz norm if and only if the absolute value function is nonexpansive (i.e. $\||x|-|y|\| \leqq\|x-y\|$ for all $x, y \in E$ ). 
Proof. Suppose the absolute value map is nonexpansive, If $|x| \leqq|y|$ then

$$
\begin{aligned}
\|x\| & =\left\|\left|x^{+}\right|-\left|-x^{-}\right|\right\| \leqq\left\|x^{+}-\left(-x^{-}\right)\right\|=\||x|\| \\
& =\frac{1}{2}\||| x|+| y||-|| x|-| y||\| \text { since }|x|-|y| \leqq 0 \\
& \leqq \frac{1}{2}|(|x|+|y|)-(|x|-|y|)\|=\|| y|\|=\|| y|-| 0 \mid\|\leqq\| y-0 \| .
\end{aligned}
$$

So $\|x\| \leqq\|y\|$, and we have a Riesz norm.

The converse follows from the inequality.

$$
|| x|-| y|| \leqq|x-y|
$$

Theorem 5. If $E$ is a vector lattice, equipped with some norm, then the following statements are equivalent.

(i) All of the lattice operations are uniformly continuous.

(ii) The absolute value function is uniformly continuous.

(iii) The absolute value function is Lipschitz continuous.

(iv) There is an absolute constant $K$ such that $\|x\| \leqq K\|y\|$ whenever $|x| \leqq|y|$.

(v) There is an equivalent Riesz norm for $E$.

Proof. (i) $\Rightarrow$ (ii). This is trivial.

(ii) $\Rightarrow$ (iii). The absolute value function is positive homogeneous.

(iii) $\Rightarrow$ (iv). This follows from the proof of Theorem 4 .

(iv) $\Rightarrow$ (v). Define $\||\cdot|\|$ by $|\|x \mid\|=\sup \{\|y\|: 0 \leqq y \leqq|x|\}$.

Statement (iv) ensures that intervals are bounded, that $\|\cdot \cdot\|$ is well defined and that \|\|$x\|\mid \leqq K\| x \|$ for all $x$. Furthermore $|x| \leqq\|x\|$ implies that $\|x\| \leqq K\||x|\| \leqq K\||x|\|$ and thus IIf $\mid \|$ in equivalent' to $\|\cdot\|$. Subadditivity of $\|\cdot\| \cdot \|$ follows from the Riesz decomposition property. Finally it is clear that $\|\cdot|\||$ is absolute.

(v) $\Rightarrow$ (i). The inequality $\left|x^{+}-y^{+}\right| \leqq|x-y|$ shows that $x \rightarrow x^{+}$is uniformly continuous with respect to any Riesz norm, hence uniformly continuous with respect to the given norm. The same is true for the other lattice operations.

An approximate version of Theorem 1 is not so satisfactory. The proof of Theorem 1 gives the following result: suppose that every order interval is bounded, and that for some constant $k<2$, we have $\|x\| \leqq k\||x|\|$ for all $x \in E$. Then $\|y\|<k(2-k)^{-1}\|x\|$ whenever $|y| \leqq x$. If in addition we assume that $\||x|\| \leqq K\|x\|$ for all $x$, we then have $\|y\| \leqq k K(2-k)^{-1}\|x\|$ whenever $|y| \leqq|x|$. But if $\mathbb{R}^{2}$ has the usual order, and the norm $\|(x, y)\|^{2}=(x+y)^{2}+4(x-y)^{2}$, we see that there may be an equivalent Riesz norm even in the case $k \geqq 2$.

In view of Theorem 5, it is natural to ask if an absolute norm, with respect to which the lattice operations are continuous, is automatically a Riesz norm? The next example shows that this is not so. First, we need a technical result.

Proposition 6. Let $E$ be a vector lattice, equipped with a norm which makes it a reflexive Banach space. 
(i) If the positive cone $K$ is closed and the lattice operations are norm continuous at the origin, then they are norm-to-weak continuous.

(ii) If, in addition to (i) the norm is absolute and has the Kadec-Klee property, then the lattice operations are norm-to-norm continuous.

(iii) The lattice operations are weakly sequentially continuous if and only if they are both norm and weakly sequentially continuous at the origin, and $K$ is closed.

(iv) Suppose $E$ is smooth, so that there is a unique support map $x \rightarrow f_{x}: E \rightarrow E^{*}$. (The support map is determined by $\left\|f_{x}\right\|=\|x\|$ and $f_{x}(x)=\|x\|^{2}$.) Suppose that the support map, restricted to $K$, is weakly sequentially continuous at the origin. If $K$ is closed, and $\||x|\| \leqq\|x\|$ for all $x$, then the lattice operations are norm continuous.

Proof. (i) Suppose that $x_{n} \rightarrow x$. Then $\left|x_{n}-x\right| \rightarrow 0$. If $f \in E^{*}$ is a positive functional, then

$$
\left|f\left(\left|x_{n}\right|-|x|\right)\right|=f\left( \pm\left(\left|x_{n}\right|-\left|x^{4}\right|\right)\right) \leqq f\left(\left|x_{n}-x\right|\right) \rightarrow 0 \text {. }
$$

Thus $f\left(\left|x_{n}\right|\right) \rightarrow f(|x|)$. Since $K$ is closed, the positive linear functionals separate the points of $E$. (If $-y \notin K$, the Hahn Banach theorem gives us a linear map $f: E \rightarrow \mathbb{R}$ satisfying $f(y)$ $=d(y,-K) \neq 0$ and $f(z) \leqq d(z,-K)$ for all $z$. Clearly $f(K) \geqq 0$ and $\|f\| \leqq 1$.) It follows from reflexivity that the linear span of the positive functionals is norm dense in $E^{*}$. Since the sequence $\left(\left|x_{n}\right|\right)$ is bounded, we may conclude that $\left|x_{n}\right| \rightarrow|x|$ weakly.

(ii) The Kadec-Klee property means that $x_{n} \rightarrow x$ whenever $x_{n} \rightarrow x$ weakly and $\left\|x_{n}\right\| \rightarrow\|x\|$. Thus (ii) is immediate from (i).

(iii) Sufficiency of the conditions follows from the proof of (i). If the lattice operations are weakly sequentially continuous, then $\{|x|:|x| \mid \leqq 1\}$ is a weakly sequentially compact, hence bounded, subset of $E$. It follows that the lattice operations are norm continuous at the origin. Necessity is now clear.

(iv) The condition $\||x|\| \leqq\|x\|$ implies that, for any $\lambda \geqq 0$,

$$
\left\|x^{+}+\lambda x^{-}\right\|-\left\|x^{+}\right\| \leqq\left\|x^{+}-\lambda x^{-}\right\|-\left\|x^{+}\right\| .
$$

It follows that $f_{x^{+}}\left(x^{-}\right) \leqq f_{x^{+}}\left(-x^{-}\right)$and so $\left\|x^{+}\right\|^{2}=f_{x^{+}}\left(x^{+}\right) \leqq f_{x^{+}}(x)$ for all $x \in E$. We first prove that if $y \geqq 0$ and $h_{n} \rightarrow 0$ then $y \wedge h_{n} \rightarrow 0$. From part (i) we know that $y \wedge h_{n} \rightarrow 0$ weakly. If $x_{n}=h_{n}-y$ then $x_{n}^{+}=h_{n}-y \wedge h_{n} \rightarrow 0$ weakly. $f_{x_{n}^{+}} \rightarrow 0$ weakly and $x_{n} \rightarrow y$ and so $\left\|x_{n}^{+}\right\|^{2} \leqq f_{x_{n}}\left(x_{n}\right) \rightarrow 0$. Thus $y \wedge h_{n}=h_{n}-x_{n}^{+} \rightarrow 0$, as required. The identity

$$
x^{+}-(x+h)^{+}=x^{+} \wedge h^{-}+x^{-} \wedge h^{+}-h^{-}
$$

now shows that $\left(x+h_{n}\right)^{+} \rightarrow x^{+}$whenever $h_{n} \rightarrow 0$. This implies that all of the lattice operations are norm continuous.

Corollary 7. Suppose $E=l_{p}(S)$, for $1<p<\infty$, or that $E$ is a Hilbert space, equipped with the usual norm. Suppose that some closed cone $K$ induces a lattice ordering on $E$. If $\||x|\| \leqq\|x\|$ for all $x$, then the lattice operations are continuous.

Proof. It is routine to show that the support map is weakly sequentially continuous at the origin. 
Note that in $l_{p}(p \neq 2)$ the support map is weakly continuous only at the origin. The partial sum cone in $l_{p}$, defined by $K=\left\{\left(x_{k}\right)_{k=1}^{\infty}: \sum_{k=1}^{n} x_{k} \geqq 0\right.$ for all $\left.n\right\}$ satisfies the hypotheses of Corollary 7 , and so has continuous lattice operations.

The lexicographic order on $\mathbb{R}^{n}$ shows closedness of the cone to be an essential assumption in Corollary 7.

Example 2. Choose $p \in(1, \infty)$. Let $E$ be the space of absolutely continuous functions $f:[0,1] \rightarrow \mathbb{R}$ such that $f(0)=0$ and $f^{\prime} \in L_{p}([0,1])$. Equipped with the pointwise order, $E$ is a vector lattice. With the norm $\|f\|=\left(\int\left|f^{\prime}\right| p\right)^{1 / p}$, it is a reflexive Banach space. As in Example 1, the norm is absolute, but the interval $[0,1]$ is unbounded. Thus no equivalent norm on $E$ can be a Riesz norm. Despite this, the lattice operations, by Proposition 6 (ii), are continuous. (By Theorem 5, they cannot be uniformly continuous.)

Clearly $E$ may be identified with $L_{p}([0,1])$ and the positive cone with $K=\left\{f: \int_{0}^{t} f \geqq 0\right.$ for all $t \in[0,1]\}$. Then $E^{*}=L_{q}([0,1])$ where $q=p /(p-1)$ and the dual cone, $K^{0}$, is the set of positive decreasing functions in $L_{q}([0,1])$. This cone does not generate $E^{*}$, but it does induce a lattice ordering on its linear span, which is dense in $E^{*}$. If we define $f(t)$ $=t(t \in[0,1])$ then with respect to this ordering, $|f|(t)=2-t$. Indeed, $g \geqq f \Rightarrow g(1) \geqq f(1)$ and for $t \leqq 1, g \geqq-f \Rightarrow g(t)+f(t) \geqq g(1)+f(1)$. It is now evident that the dual norm is not absolute.

A similar argument shows that the lattice operations are continuous in any Sobolev space $W_{1, p}(\Omega)$ for $1<p<\infty$ and $\Omega$ an open set in $\mathbb{R}^{n}$. This can also be proved directly for $1 \leqq p<\infty$. Slightly more care is needed to show that the norm is absolute if $n \geqq 2$. For $m \geqq 2$, the pointwise order on $W_{m, p}(\Omega)$ is not a lattice order.

\section{Hilbert lattices}

Let $E$ be a vector lattice with positive cone $K$. Equip $E$ with an inner product $\langle$, and its corresponding norm. We investigate the relationship between these two structures, and their different notions of orthogonality.

Theorem 8. Let $E$ be a Hilbert space, lattice-ordered by a closed cone $K$.

Consider the following properties that $E$ may or may not have:

(1) For any $x, y \in E$ we have $x \wedge y=0$ if and only if $x \geqq 0, y \geqq 0$ and $\langle x, y\rangle=0$.

(1*) The norm is a Riesz norm.

(2) For any $x, y \in E$ with $x \wedge y=0$, we have $\langle x, y\rangle=0$.

(2*) The norm is absolute.

(3) $K$ is contained in the dual cone $K^{0}=\{x:\langle x, y\rangle \geqq 0, y \in K\}$.

$\left(^{*}\right)$ The norm is absolutely monotone, i.e. $\|x\| \leqq\|y\|$ whenever $|x| \leqq y$.

(4) The dual cone $K^{0}$ is contained in $K$.

(4*) For any $x \in E$, we can find $y \geqq|x|$ with $\|y\| \leqq\|x\|$.

(5) For any $x, y \in E$ with $x \wedge y=0$, we have $\langle x, y\rangle \leqq 0$.

$\left(5^{*}\right)$ For any $x \in E$ we have $\||x|\| \leqq\|x\|$. 
Then property $(n)$ is equivalent to property $\left(n^{*}\right)$, for $n=1,2,3,4,5$. Furthermore $(1) \Rightarrow(2) \Rightarrow(5) \Rightarrow(4)$ and so property (1) holds if any only if (3) and (n) both hold, for $n=2,4$ or 5. In particular, $\|\cdot\|$ is a Riesz norm if and only if $K^{0}=K$.

Proof. $(5) \Rightarrow\left(5^{*}\right)$ For any $x$ we have $x^{+} \wedge x^{-}=0$ and so $\left\langle x^{+}, x^{-}\right\rangle \leqq 0$. It follows that $\left\|x^{+}+x^{-}\right\|^{2} \leqq\left\|x^{+}-x^{-}\right\|^{2}$.

$\left(5^{*}\right) \Rightarrow(5)$ if $x \wedge y=0$ and $z=x-y$ then $|z|=x+y$. Hence $\|x+y\|^{2} \leqq\|x-y\|^{2}$ and so $\langle x, y\rangle \leqq 0$.

$(4) \Rightarrow\left(4^{*}\right)$ This is the only part of the proof which requires $E$ to be complete or $K$ to be closed. Fix $x \in E$ with $\|x\|=1$. Then $S=\left\{v\right.$ : there exists $w$ with $v+w, v-w \in K^{0}$ and $\langle x, w\rangle=1\}$ is a convex set. If $v \in S$ with $w$ as above then $v+w \in K^{0}, v-w \in K$ and $1=\langle x, w\rangle^{2} \leqq\|x\|^{2}\|w\|^{2} \leqq\|w\|^{2}+\langle v+w, v-w\rangle=\|v\|^{2}$. Thus $S$ is disjoint from the open unit ball of $E$. The separation theorem then gives us $y \in E$ with $\langle y, u\rangle<1$ whenever $\|u\|<1$ and $\langle y, v\rangle \geqq 1$ for all $v \in S$. Consider any $z \in K^{0}$ with $\langle x, z\rangle>0$. If $v=\langle x, z\rangle^{-1} z$ it is easy to see that $v \in S$. Thus $\langle y, v\rangle \geqq 1$ i.e. $\langle y, z\rangle \geqq\langle x, z\rangle$. Clearly $\langle y, z\rangle \geqq-\langle x, z\rangle$ also. If $\langle x, z\rangle\left\langle 0\right.$, put $v=-\langle x, z\rangle^{-1} z$. The same argument shows that $\langle y, z\rangle \geqq-\langle x, z\rangle \geqq\langle x, z\rangle$. It follows that $\langle y \pm x, z\rangle \geqq 0$ for all $z \in K^{0}$ and so $y \pm x \in K^{00}$. Since $K$ is closed, the bipolar theorem tells us $y \geqq \pm x$. It is clear that $\|y\| \leqq 1$.

$\left(4^{*}\right) \Rightarrow(4)$. Let $x \in K^{0}$. Then we can find $y \geqq \pm x$ with $\|y\| \leqq\|x\|$. Then $y-x \in K$, so $\|x\|^{2} \geqq\|x\|\|y\| \geqq\langle x, y\rangle=\langle x, y-x\rangle+\|x\|^{2} \geqq\|x\|^{2}$. Hence $\|x\|^{2}=\|y\|^{2}=\langle x, y\rangle$ and $\|y-x\|^{2}=\|y\|^{2}+\|x\|^{2}-2\langle x, y\rangle=0$. Thus $x=y \in K$.

(3) $\Rightarrow\left(3^{*}\right)$. Suppose $-y \leqq x \leqq y$. Then $y-x \in K$ and $y+x \in K \subseteq K^{0}$. By assumption $\langle y+x, y-x\rangle \geqq 0$, which is the assertion as $\|y\|^{2} \geqq\|x\|^{2}$.

$\left(3^{*}\right) \Rightarrow(3)$. Let $x \in K$. Then, for any $y \in K$, we have $-(x+y) \leqq x-y \leqq x+y$ and so $\| x$ $-y\|\leqq\| x+y \|$, i.e. $\langle x, y\rangle \geqq 0$. Thus $x \in K^{0}$.

$(2) \Rightarrow\left(2^{*}\right)$. For any $x \in E$, we have $x=x^{+}-x^{-}$where $x^{+} \wedge x^{-}=0$. Thus $\left\langle x^{+}, x^{-}\right\rangle=0$ and so $\left\|x^{+}+x^{-}\right\|^{2}=\left\|x^{+}-x^{-}\right\|^{2}$, i.e. $\||x|\|=\|x\|$.

$\left(2^{*}\right) \Rightarrow(2)$. Suppose $x \wedge y=0$. If $z=x-y$ then $z^{+}=x$ and $z^{-}=y$. Hence $\|x+y\|=\||z|\|$ $=\|z\|=\|x-y\|$, and so $\langle x, y\rangle=0$.

$(1) \Rightarrow(2) \&(3)$. It is obvious that $(1) \Rightarrow(2)$. Suppose (3) fails. Then $\langle x, y\rangle<0$ for some $x, y \in K$. Choose $\lambda \in(0,1)$ so that $\langle x, z\rangle=0$, where $z=\lambda x+(1-\lambda) y$. Then $z \geqq 0$ and $x \wedge z \geqq \lambda x$ so $x \wedge z \neq 0$. This contradicts (1).

(2) \& (3) $\Rightarrow(1)$. Suppose $x \geqq 0, y \geqq 0$ and $\langle x, y\rangle \leqq 0$. We must show that $x \wedge y=0$. Put $z=x \wedge y$. Then $(x-z) \wedge(y-z)=0$ and (2) tells us that $\langle x-z, y-z\rangle=0$. Since $0 \leqq z \leqq x, y$, we deduce from (3) that $\|z\|^{2} \leqq\langle z, z\rangle+\langle x+y-2 z, z\rangle=\langle x, y\rangle-\langle x-z, y-z\rangle=0$. So $z=0$, as required.

$(1) \Leftrightarrow\left(1^{*}\right)$. This is now clear, since $\left(1^{*}\right) \Leftrightarrow\left(2^{*}\right) \&\left(3^{*}\right) .(1) \Rightarrow(2) \Rightarrow(5) \Rightarrow(4)$. Obviously $(1) \Rightarrow(2) \Rightarrow(5) \Rightarrow\left(5^{*}\right) \Rightarrow\left(4^{*}\right)$. (1) $\Leftrightarrow(3)$ and $(4) \Leftrightarrow(3)$ and (5). It is now clear that $(1) \Rightarrow(3)$ and $(5) \Rightarrow(3)$ and (4). To finish the proof, we show that $\left(3^{*}\right)$ and $\left(4^{*}\right) \Rightarrow\left(2^{*}\right)$. Given $x,\left(3^{*}\right)$ tells us that $\|x\| \leqq\||x|\|$. If $y$ is given by $\left(4^{*}\right)$ then $\|y\| \leqq\|x\| \leqq\||x|\| \leqq\|y\|$, the last inequality holding by $\left(3^{*}\right)$. Thus the norm is absolute.

One can also show that the property $\|x\| \leqq\||x|\|$ for all $x$ is equivalent to $x \wedge y$ $=0 \Rightarrow\langle x, y\rangle \geqq 0$. However, this property does not seem to be of much use.

Schaefer [2, p. 270] calls any space satisfying (1) a Hilbert lattice, and shows that any Hilbert lattice is isomorphic to some $L_{2}(S)$, equipped with the pointwise order.

The only implications (between the above five properties) which always hold are those 
which follow immediately from Theorem 8. Example 2 (with $p=2$ ) shows that (2) does not imply (3). Simple two-dimensional examples (or alternatively Example 3) show that (3) does not imply (4) and Example 5 shows that (4) does not imply (5). Another twodimensional example (or Example 4) shows that (5) does not imply (2). The next two examples are designed to show that, without absoluteness, continuity of the lattice operations is unrelated to boundedness of intervals.

Example 3. Let $l_{2}$ be equipped with the standard inner product and the ordering given by the positive cone $K=\left\{\left(x_{k}\right)_{k=1}^{\infty}: x_{2 k-1} \geqq k x_{2 k} \geqq 0\right.$ for all $\left.k\right\}$. This is a lattice ordering on $E=K-K$; the join, for example, is given by

$$
\begin{gathered}
(x \vee y)_{2 k}=x_{2 k} \vee y_{2 k} \text { and } \\
(x \vee y)_{2 k-1}=k(x \vee y)_{2 k}+\left(x_{2 k-1}-k x_{2 k}\right) \vee\left(y_{2 k-1}-k y_{2 k}\right) .
\end{gathered}
$$

This norm is monotone, since $K$ is contained in the standard cone for $l_{2}$. However if

$$
x_{n}=\frac{1}{n}\left(e_{2 n}-e_{2 n-1}\right)
$$

then $x_{n} \rightarrow 0$ (in norm) but

$$
\left|x_{n}\right|=\frac{1}{n} e_{2 n}+\left(2+\frac{1}{n}\right) e_{2 n-1},
$$

so $\left\|\left|x_{n}\right|\right\| \geqq 2$. The lattice operations are not even continuous at the origin, so there can be no equivalent Riesz norm.

Example 4. Let $E=l_{2}$, but ordered by the positive cone $K=\left\{\left(x_{k}\right)_{k=1}^{\infty}: x_{2 k}-1 \geqq 0\right.$, $x_{2 k} \geqq k x_{2 k-1}$ for all $\left.k\right\}$. This is also a lattice ordering, with the lattice operations given by

$$
\begin{gathered}
(x \vee y)_{2 k-1}=x_{2 k-1} \vee y_{2 k-1} \\
(x \vee y)_{2 k}=\left(x_{2 k}+k x_{2 k-1}\right) \vee\left(y_{2 k}+k y_{2 k-1}\right)-k(x \vee y)_{2 k-1}
\end{gathered}
$$

If we put $x_{2 k-1}=x_{2 k}=k^{-2 / 3}$ then $x \in E$ but the interval $[0, x]$ is unbounded. In fact if $y_{n}=n^{-2 / 3} e_{2 n-1}-n^{1 / 3} e_{2 n}$ then $0 \leqq y_{n} \leqq x$ for all $n$, but $\left\|y_{n}\right\| \geqq n^{1 / 3}$ is unbounded. If we put

$$
x_{n}=\frac{1}{2 n} e_{2 n-1}-e_{2 n} \text { then }\left|x_{n}\right|=\frac{1}{2 n} e_{2 n-1}
$$

and so $\left|x_{n}\right| \rightarrow 0$ yet $\left\|x_{n}\right\| \geqq 1$. Thus no equivalent norm on $E$ can be absolute.

Despite this, the lattice operations are continuous. To see this, first note that $(|y+k x|$ $-|k x|)^{2} \leqq|y|^{2}$ for any $x, y \in R$. Thus $|\cdot|$ is norm decreasing. Since $K$ is a closed cone, Corollary 7 gives us the required continuity. It is also possible, but tedious, to verify directly that $|\cdot|$ is continuous. 
We remark that Examples 3 and 4 are dual to one another, under the natural pairing $\langle x, y\rangle=\sum_{k=1}^{\infty} x_{k} y_{k}$.

Example 5. Let $E=\mathbb{B}^{3}$ be equipped with the usual euclidean norm, and ordered by the positive cone $K=\{(x, y, z): x \geqq 0, x+y \geqq 0, x-y+z \geqq 0\}$. Then the extreme rays of $K^{0}$ are $\mathbb{R}(1,0,0), \mathbb{R}(1,1,0)$ and $\mathbb{R}(1,-1,1)$ whence $K^{0} \subset K$. If $x=(0,1,0) \in E$ then, with respect to this ordering, we have $|x|=(0,1,2)$. This shows that we cannot take $y=|x|$ in property $\left(4^{*}\right)$ of Theorem 8 .

Acknowledgements. The authors are grateful to J. J. Schäffer for pointing out that a norm on a function space, defined in terms of the derivative as in Example 2, will be absolute but not monotone. The second author is grateful to Dalhousie University, for hospitality and financial support during his visit.

\section{REFERENCES}

1. F. L. Bauer, J. Stoer and C. WitzGall, Absolute and monotonic norms, Numer. Math. 3 (1961), 257-264.

2. H. H. SCHAEFER, Banach lattices and positive operators (Springer-Verlag, Berlin, 1974).

Department of Mathematics, Statistics

And Computing SCIEnce

Dalhousie UNiversity

Halifax, Nova Scotia

CANADA B3H $4 \mathrm{H} 8$
Department of Mathematics Institute of Advanced STUdies Australian National University Canberra, Australia 